\title{
Intermittent Control for Cluster-Delay Synchronization in Directed Networks
}

\author{
Jianbao Zhang $\mathbb{D}^{1,2,3}$ Yi Wang, ${ }^{4}$ Zhongjun Ma $\mathbb{D}^{5},{ }^{5}$ Jianlong Qiu, ${ }^{3,6}$ and Fawaz Alsaadi ${ }^{6}$ \\ ${ }^{1}$ School of Information Science and Technology, Linyi University, Linyi 276005, China \\ ${ }^{2}$ School of Automation, Southeast University, Nanjing 210096, China \\ ${ }^{3}$ Key Laboratory of Complex Systems and Intelligent Computing in Universities of Shandong (Linyi University), Linyi 276005, China \\ ${ }^{4}$ School of Data Sciences, Zhejiang University of Finance and Economics, Hangzhou 310018, China \\ ${ }^{5}$ School of Mathematics and Computing Science, Guilin University of Electronic Technology, Guilin 541004, China \\ ${ }^{6}$ Department of Information Technology, King Abdulaziz University, Jeddah 21589, Saudi Arabia
}

Correspondence should be addressed to Zhongjun Ma; mzj1234402@163.com

Received 30 September 2017; Revised 8 January 2018; Accepted 24 January 2018; Published 18 February 2018

Academic Editor: Imre J. Rudas

Copyright (c) 2018 Jianbao Zhang et al. This is an open access article distributed under the Creative Commons Attribution License, which permits unrestricted use, distribution, and reproduction in any medium, provided the original work is properly cited.

\begin{abstract}
We investigate cluster-delay synchronization of a directed network possessing cluster structures by designing an intermittent control protocol. Based on Lyapunov stability theory, we proved that synchronization can be realized for oscillators in the same cluster and cluster-delay synchronization can be realized for the whole network. By simplifying the obtained sufficient conditions, we carry out a succinct and utilitarian corollary. In addition, comparative researches are carried out to show the differences and the usefulness of the obtained results with respect to other similar controllers from the recent literature. Finally we provide two numerical examples to show the effectiveness of the control schemes.
\end{abstract}

\section{Introduction}

Chaos theory is an interdisciplinary theory studying the unstable aperiodic behavior of dynamical systems. The most distinguishing characteristic of chaotic systems is the highly sensitive dependence on initial conditions [1], which implies that even if the present determines the future, the approximate present does not approximately determine the future. Therefore, it is difficult to control an unpredictable chaotic system in the long term. However, due to the wide applications of chaos theory, more and more researchers are devoting themselves to studying chaos control theory in many fields of science and engineering.

In 1990, it was discovered surprisingly that two chaotic systems started from different initial conditions could synchronize with each other [2], and the great discovery immediately attracted lots of attention and became an important issues of chaos control. Since then, several effective methods have been applied to study synchronization of chaos oscillators. In 1998, the famous master stability function method was proposed to study the local stability of the synchronous state [3], which is based on the calculation of the maximum Lyapunov exponent for the least stable transversal mode of the synchronous manifold and the eigenvalues of the connection matrix. Later, Lyapunov function method was employed to investigate global stability of the synchronous state [4]. Based on the two methods mentioned above, many other surveys have been carried out to explore the mysterious mechanisms of chaos synchronization and chaos control. As the study develops in depth, various kinds of synchronization protocols have been put forward and deeply studied, such as complete synchronization [5], exponential synchronization $[6,7]$, projective synchronization $[8,9]$, lag synchronization [10], and cluster synchronization [11-13]. The above-mentioned results only discussed synchronization induced by mutual coupling and the intrinsic structure of the network.

During the past decades, many external control strategies have been carried out to synchronize complex networks, such as adaptive control [14, 15], impulsive control [16], sliding 
mode control [17], pinning control [18-20], sliding mode control [21], and intermittent control [22, 23]. Those control strategies have been widely investigated and used in many network control problems. The primary concern of this paper is cluster synchronization under external control, which has attracted widespread attention [24,25]. By designing adaptive pinning-control schemes on both coupling strengths and feedback gains, it was shown that a network can realize cluster synchronization under weak coupling strengths and small feedback gains [15]. Later, another feedback controller was designed to realize cluster synchronization under the condition that the topology of each cluster has a directed spanning tree $[25,26]$. All in all, great efforts have been devoted to the investigation of cluster synchronization under external control. It has been shown to be an effective method to control a complex network to a desired synchronized state.

Up to now, to the best of our knowledge, there are few results concerning cluster-delay synchronization, which is a special type of collective behavior between complete synchronization and cluster synchronization. In our opinion, clusterdelay synchronization implies that the nodes in a complex network are split into several clusters, and all the nodes in the same cluster behave in a synchronous fashion, but nodes in different clusters follow distinct time evolutions with different time delays. It is a new type of collective behavior in complex networks worthy of detailed investigation, and this paper studies cluster-delay synchronization of a complex network via pinning control with intermittent effect. To achieve cluster lag synchronization in community networks, $\mathrm{Wu}$ and $\mathrm{Fu}$ designed several linear pinning controllers in view of lower cost and more convenient implementation [23]. Recently, motivated by the interesting investigation, we provided some primary theoretical analyses and numerical experiment [27]. Different from the previous results [23, 27], this paper proposes a leader-following system and derives sufficient conditions for cluster-delay synchronization via pinning control with intermittent effect. We first prove that all the oscillators in the same cluster synchronize with each other and then prove that the oscillators in different clusters behave in a synchronous mode but with different time delays. Numerical simulations show that the modified pinning-control scheme works effectively and serves different purposes in practice.

The rest of this paper is organized as follows. Section 2 introduces some necessary preliminaries and builds a modified clustered network model with an intermittent leaderfollowing controller. Then, both cluster synchronization and cluster-delay synchronization of the network model are investigated through Lyapunov theory in Section 3. Comparative researches with respect to previous controllers are also given there. In Section 4, two examples of numerical simulations are carried out to show the validity of the proposed control schemes. Finally, the main results of this paper are briefly summarized in Section 5.

\section{Preliminaries}

In this section, we make some mathematical preparations for the oscillator network model. Suppose the topology structure of the communication network is represented by a directed graph $\mathscr{G}=\{\mathscr{V}, \mathscr{E}\}$, which is composed of a set of nodes $\mathscr{V}=\{1, \ldots, N\}$ and a set of edges $\mathscr{E}=\mathscr{V} \times \mathscr{V}$. The graph exhibits a clustered structure, which implies that the $N$ nonidentical oscillators are divided into $n$ nonempty subsets called clusters. Let $\mathscr{V}_{k}=\left\{m_{k-1}+1, \ldots, m_{k}\right\}$ denote the index set of all the nodes in the $k$ th cluster, where $k=1,2, \ldots, n$, $m_{0}=0, m_{n}=N$. For convenience, we define a function $\varphi$ : $\{1, \ldots, N\} \rightarrow\{1, \ldots, n\}$, where $\varphi(i)=k$ implies that the node $i \in \mathscr{V}_{k}$.

Let $x_{i}(t)=\left(x_{i 1}(t), \ldots, x_{i d}(t)\right)^{\top}$ be the state variable of the $i$ th oscillator, the state equations of the network are given by

$$
\begin{array}{r}
\dot{x}_{i}(t)=f\left(x_{i}(t)\right)+\sum_{l=1}^{n} \sum_{j \in \mathscr{V}_{l}} a_{i j} x_{j}(t)+u_{i}(t), \\
i=1,2, \ldots, N,
\end{array}
$$

where $t \in[0,+\infty)$ is a continuous time, $f(\cdot)$ is a continuous function that describes the local dynamics of each node, $A=\left(a_{i j}\right)_{N \times N}$ is the coupling matrix with $a_{i j} \geq 0$ for $i \neq j$, and $\sum_{j=1}^{N} a_{i j}=0$ for $i=1,2, \ldots, N$. Denoting the state variables of the oscillators in $k$ th cluster as $X_{k}(t)=$ $\left(x_{m_{k-1}+1}^{\top}(t), \ldots, x_{m_{k}}^{\top}(t)\right)^{\top}, U_{k}(t)=\left(u_{m_{k-1}+1}^{\top}(t), \ldots, u_{m_{k}}^{\top}(t)\right)^{\top}$, and $F\left(X_{k}(t)\right)=\left(f\left(x_{m_{k-1}+1}(t)\right)^{\top}, \ldots, f\left(x_{m_{k}}(t)\right)^{\top}\right)^{\top}$, the network (1) can be rewritten as follows:

$$
\begin{array}{r}
\frac{d X_{k}(t)}{d t}=F\left(X_{k}(t)\right)+\sum_{l=1}^{n}\left(A_{k l} \otimes I_{d}\right) X_{l}(t)+U_{k}(t), \\
k=1,2, \ldots, n,
\end{array}
$$

where $A_{k l} \in R^{\left(m_{k}-m_{k-1}\right) \times\left(m_{l}-m_{l-1}\right)}$ are defined by the block matrix

$$
A=\left[\begin{array}{cccc}
A_{11} & A_{12} & \cdots & A_{1 n} \\
A_{21} & A_{22} & \cdots & A_{2 n} \\
\cdots & \cdots & \cdots & \cdots \\
A_{n 1} & A_{n 2} & \cdots & A_{n n}
\end{array}\right] .
$$

For convenience, we decompose matrix $A$ into two matrices as follows:

$$
A=\bar{A}+\widetilde{A},
$$

where

$$
\begin{aligned}
\bar{A} & =\left\{\bar{A}_{k l}\right\}_{n \times n}=\left\{\bar{a}_{i j}\right\}_{N \times N} \\
& = \begin{cases}0, & \varphi(i) \neq \varphi(j) ; \\
a_{i j}, & \varphi(i)=\varphi(j), i \neq j ; \\
-\sum_{j=1, j \neq i}^{N} \bar{a}_{i j}, & i=j\end{cases}
\end{aligned}
$$


representing the intracluster couplings, and

$$
\begin{aligned}
\widetilde{A}=\left\{\widetilde{A}_{k l}\right\}_{n \times n}=\left\{\widetilde{a}_{i j}\right\}_{N \times N} \\
= \begin{cases}0, & \varphi(i)=\varphi(j), i \neq j ; \\
a_{i j}, & \varphi(i) \neq \varphi(j) ; \\
-\sum_{j=1, j \neq i}^{N} \tilde{a}_{i j}, & i=j\end{cases}
\end{aligned}
$$

representing the intercluster couplings.

The dynamics of the virtual leaders in the oscillator network are described by

$$
\begin{aligned}
\dot{s}_{k}(t)=f\left(s_{k}(t)\right)-\theta_{k}(t)\left(s_{k}(t)-s_{1}\left(t-\tau_{k}\right)\right), & \\
& k=1,2, \ldots, n,
\end{aligned}
$$

where $s_{k}(t)$ is the state variable of the $k$ th virtual leader, $\tau_{k}$ is the time delay, and

$$
\theta_{k}(t)= \begin{cases}\Theta_{k}, & t \in[m T,(m+\zeta) T) ; \\ 0, & t \in[(m+\zeta) T,(m+1) T),\end{cases}
$$

where $\Theta_{k}$ is a positive constant representing the feedback control gain, $\zeta \in(0,1)$ is the control width, $k=1,2, \ldots, n$, $m=0,1,2, \ldots$. In this paper, we suppose that the time delay $\tau_{1}=0$, which implies that $\dot{s}_{1}(t)=f\left(s_{1}(t)\right)$. Different from the previous result on cluster lag synchronization [23], the leader systems in this paper are not isolated nodes $\dot{s}_{k}(t)=$ $f\left(s_{k}(t)\right)$. Instead, linear control laws are designed for the leader systems to realize cluster-delay synchronization. This paper aims to make the leader of the $k$ th cluster track the trajectory of the leader of the first cluster with a time delay $\tau_{k}, k=1, \ldots, n$.

Analyzing systems (2) and (7) comprehensively, we design the control inputs for system (2):

$$
\begin{aligned}
U_{k}(t)= & \theta_{k}(t) \iota_{m_{k}-m_{k-1}} \otimes\left[s_{k}(t)-s_{1}\left(t-\tau_{k}\right)\right] \\
& -\sum_{l=1}^{n}\left(\widetilde{A}_{k l} \otimes I_{d}\right) X_{l}(t) \\
& -\sigma_{k}\left[X_{k}(t)-l_{m_{k}-m_{k-1}} \otimes s_{1}\left(t-\tau_{k}\right)\right],
\end{aligned}
$$

where the vector $\iota_{m_{k}-m_{k-1}}=(1, \ldots, 1)^{\top} \in R^{m_{k}-m_{k-1}}$; the constant $\sigma_{k} \geq 0$ is the feedback control gain; $T>0$ is the control period; $k=1,2, \ldots, n$.

\section{Main Results}

In this section, we will derive some sufficient conditions for both cluster synchronization and cluster-delay synchronization. Before that, it is necessary to introduce the following assumptions.

Assumption 1. There exists a positive constant $\delta$ such that the vector function $f$ satisfies that

$$
(x-y)^{T}(f(x)-f(y)) \leq \delta(x-y)^{T}(x-y)
$$

for any $x, y \in R^{d}$.
It has been checked that many well-known chaotic systems, such as cellular neural networks, Lorenz system, Chen system, Rössler system, and Chua's circuit, satisfy Assumption $1[28,29]$.

3.1. Cluster Synchronization Analysis. Now, we first introduce the following definition of both cluster synchronization and cluster-delay synchronization [27].

Definition 2. Define the synchronization errors $e_{i}(t)=$ $x_{i}(t)-s_{\varphi(i)}(t), E_{k}(t)=X_{k}(t)-\iota_{m_{k}-m_{k-1}} \otimes s_{k}(t), E(t)=$ $\left(E_{1}^{\top}(t), \ldots, E_{n}^{\top}(t)\right)^{\top}, e_{k}^{\tau}(t)=s_{k}(t)-s_{1}\left(t-\tau_{k}\right), E^{\tau}(t)=$ $\left(e_{1}^{\tau^{\top}}(t), \ldots, e_{n}^{\tau^{\top}}(t)\right)^{\top}$. The oscillator network (2)-(7) is said to realize cluster synchronization, if the synchronization errors satisfy

$$
\lim _{t \rightarrow \infty} \sum_{i=1}^{N}\left\|e_{i}(t)\right\|=\lim _{t \rightarrow \infty} \sum_{k=1}^{n}\left\|E_{k}(t)\right\|=\lim _{t \rightarrow \infty}\|E(t)\|=0 .
$$

The oscillator network (2)-(7) is said to realize cluster-delay synchronization, if the synchronization errors satisfy equality (11) and

$$
\lim _{t \rightarrow \infty} \sum_{k=2}^{n}\left\|e_{k}^{\tau}(t)\right\|=\lim _{t \rightarrow \infty}\left\|E^{\tau}(t)\right\|=0 .
$$

The preliminaries above, together with Lyapunov function method, bring us to the following theorem for cluster synchronization, which implies that the $m_{k}-m_{k-1}$ oscillators in cluster $\mathscr{V}_{k}$ to synchronize with each other, $k=1,2, \ldots, d$.

Theorem 3. Suppose that Assumption 1 holds; the oscillator network (2)-(7) with the control protocol (9) realizes cluster synchronization if the matrix $D-\left(\bar{A}+\bar{A}^{\top}\right) / 2$ is negative definite, where $D=\operatorname{diag}\left\{\left(\delta-\sigma_{1}\right) I_{m_{1}-m_{0}}, \ldots,\left(\delta-\sigma_{n}\right) I_{m_{n}-m_{n-1}}\right\}$.

Proof. Noticing that $E_{k}(t)=X_{k}(t)-\iota_{m_{k}-m_{k-1}} \otimes s_{k}(t)$, we obtain the error system of the $k$ th cluster as follows:

$$
\begin{aligned}
\frac{d E_{k}(t)}{d t}= & \frac{d X_{k}(t)}{d t}-\iota_{m_{k}-m_{k-1}} \otimes \frac{d s_{k}(t)}{d t} \\
= & {\left[F\left(X_{k}(t)\right)-\iota_{m_{k}-m_{k-1}} \otimes f\left(s_{k}(t)\right)\right] } \\
& +\sum_{l=1}^{n}\left(\bar{A}_{k l} \otimes I_{d}\right) X_{l}(t) \\
& -\sigma_{k}\left[X_{k}(t)-\iota_{m_{k}-m_{k-1}} \otimes s_{1}\left(t-\tau_{k}\right)\right] \\
= & {\left[F\left(X_{k}(t)\right)-F\left(\iota_{m_{k}-m_{k-1}} \otimes s_{k}(t)\right)\right] } \\
& +\sum_{l=1}^{n}\left(\bar{A}_{k l} \otimes I_{d}\right) E_{l}(t)-\sigma_{k} E_{k}(t) .
\end{aligned}
$$

Consider the following Lyapunov function:

$$
V_{1}(t)=\frac{1}{2} \sum_{k=1}^{n} E_{k}^{\top}(t) E_{k}(t) \text {. }
$$


The derivative of $V_{1}(t)$ along the trajectories of the error systems (13) can be calculated as follows:

$$
\begin{aligned}
& \frac{d V_{1}(t)}{d t}=\sum_{k=1}^{n} E_{k}^{\top}(t) \frac{d E_{k}(t)}{d t}=\sum_{k=1}^{n} E_{k}^{\top}(t) \\
& \cdot\left[\left(F\left(X_{k}(t)\right)-F\left(l_{m_{k}-m_{k-1}} \otimes s_{k}(t)\right)\right)\right. \\
& \left.+\sum_{l=1}^{n}\left(\bar{A}_{k l} \otimes I_{d}\right) E_{l}(t)-\sigma_{k} E_{k}(t)\right] \\
& \leq \sum_{k=1}^{n}\left(\delta-\sigma_{k}\right) E_{k}^{\top}(t) E_{k}(t)+\sum_{l=1}^{n} E_{k}^{\top}(t)\left(\bar{A}_{k l} \otimes I_{d}\right) \\
& \cdot E_{l}(t)=E^{\top}(t)\left[\left(D-\frac{\left(\bar{A}+\bar{A}^{\top}\right)}{2}\right) \otimes I_{d}\right] E(t) .
\end{aligned}
$$

According to the conditions of Theorem 3 and Lyapunov stability theory, the solutions of the oscillator network satisfy that $\lim _{t \rightarrow \infty}\left\|E_{k}(t)\right\|=0$ for all $k=1,2, \ldots, n$. Hence, the oscillator network (2)-(7) with the control protocol (9) realizes cluster synchronization. The proof is completed.

Noticing that matrix $\bar{A}$ represents the intracluster couplings and matrix $\widetilde{A}$ represents the intercluster couplings, one gets that the results of Theorem 3 is irrelevant to the intercluster couplings. In other words, cluster synchronization can be guaranteed by the intracluster couplings of each cluster, and the intercluster couplings can be chosen arbitrarily.

3.2. Cluster-Delay Synchronization Analysis. Now, we are in a position to carry out the following theorem on clusterdelay synchronization, which implies that the oscillators in the same cluster behave in a synchronous fashion, but oscillators in different clusters follow distinct time evolutions with different time delays.

Theorem 4. Suppose that Assumption 1 holds, the oscillator network (2)-(7) with the control protocol (9) realizes clusterdelay synchronization if

(i) the matrix $D-\left(\bar{A}+\bar{A}^{\top}\right) / 2$ is negative definite;

(ii) there exists a positive constant $\alpha$ such that the matrix $(\delta+\alpha) I_{n}-\Theta$ is negative semidefinite, where $\Theta=$ $\operatorname{diag}\left\{\Theta_{1}, \Theta_{2}, \ldots, \Theta_{n}\right\}$;

(iii) the constant $\delta-\delta \zeta-\alpha \zeta \leq 0$.

Proof. According to Theorem 3 and condition (i) of Theorem 4, it is easy to prove that

$$
\lim _{t \rightarrow \infty} \sum_{i=1}^{N}\left\|e_{i}(t)\right\|=\lim _{t \rightarrow \infty} \sum_{k=1}^{n}\left\|E_{k}(t)\right\|=\lim _{t \rightarrow \infty}\|E(t)\|=0 .
$$

Now, we will prove that

$$
\lim _{t \rightarrow \infty} \sum_{k=2}^{n}\left\|e_{k}^{\tau}(t)\right\|=\lim _{t \rightarrow \infty}\left\|E^{\tau}(t)\right\|=0
$$

Consider the following Lyapunov function:

$$
V_{2}\left(E^{\tau}(t)\right)=\frac{1}{2} \sum_{k=1}^{n} e_{k}^{\tau^{\top}}(t) e_{k}^{\tau}(t)=\frac{1}{2} E^{\tau^{\top}}(t) E^{\tau}(t) .
$$

From the oscillator network (2)-(7) with the control protocol (9), it is easy to get the following error system:

$$
\begin{aligned}
\frac{d e_{k}^{\tau}(t)}{d t}= & \frac{d s_{k}(t)}{d t}-\frac{d s_{1}\left(t-\tau_{k}\right)}{d t} \\
= & f\left(s_{k}(t)\right)-f\left(s_{1}\left(t-\tau_{k}\right)\right)-\theta_{k}(t) e_{k}^{\tau}(t), \\
& \quad k=1,2, \ldots, n .
\end{aligned}
$$

Calculating the derivative of $V_{2}\left(E^{\tau}(t)\right)$, one obtains

$$
\begin{array}{r}
\frac{d V_{2}\left(E^{\tau}(t)\right)}{d t}=\sum_{k=1}^{n} e_{k}^{\tau^{\top}}(t) \frac{d e_{k}^{\tau}(t)}{d t}=\sum_{k=1}^{n} e_{k}^{\tau^{\top}}(t) \\
\cdot\left[f\left(s_{k}(t)\right)-f\left(s_{1}\left(t-\tau_{k}\right)\right)-\theta_{k}(t) e_{k}^{\tau}(t)\right] \\
\leq \sum_{k=1}^{n} \delta e_{k}^{\tau^{\top}}(t) e_{k}^{\tau}(t)-\sum_{k=1}^{n} \theta_{k}(t) e_{k}^{\tau^{\top}}(t) e_{k}^{\tau}(t) .
\end{array}
$$

On the interval $t \in[m T,(m+\zeta) T), m=0,1,2, \ldots$, inequality (20) can be reduced to the following form:

$$
\begin{aligned}
\frac{d V_{2}\left(E^{\tau}(t)\right)}{d t} \leq & \sum_{k=1}^{n} \delta e_{k}^{\tau^{\top}}(t) e_{k}^{\tau}(t)-\sum_{k=1}^{n} \Theta_{k} e_{k}^{\tau^{\top}}(t) e_{k}^{\tau}(t) \\
= & E^{\tau^{\top}}(t)\left[(\delta+\alpha) I_{n}-\Theta\right] \otimes I_{d} E^{\tau}(t) \\
& -\alpha E^{\tau^{\top}}(t) E^{\tau}(t) \leq-2 \alpha V_{2}\left(E^{\tau}(t)\right) .
\end{aligned}
$$

Integrating the above inequality over the interval $[m T,(m+$ $\zeta) T$ ), one has

$$
V_{2}\left(E^{\tau}(t)\right) \leq V_{2}\left(E^{\tau^{\top}}(m T)\right) e^{-2 \alpha(t-m T)} .
$$

On the interval $t \in[(m+\zeta) T,(m+1) T)$, the inequality (20) can be reduced to the following form:

$$
\frac{d V_{2}\left(E^{\tau}(t)\right)}{d t} \leq \sum_{k=1}^{n} \delta e_{k}^{\tau^{\top}} e_{k}^{\tau} \leq 2 \delta V_{2}\left(E^{\tau}(t)\right),
$$

which is equivalent to

$$
V_{2}\left(E^{\tau}(t)\right) \leq V_{2}\left(E^{\tau}((m+\zeta) T)\right) e^{2 \delta(t-(m+\zeta) T)} .
$$

Now, we will prove the following inequality by mathematical induction on parameter $m$ :

$$
\begin{gathered}
V_{2}\left(E^{\tau}(t)\right) \leq V_{2}\left(E^{\tau}(0)\right) e^{2 m(\delta+\alpha)(1-\zeta) T-2 \alpha t}, \\
m T \leq t<(m+\zeta) T ; \\
V_{2}\left(E^{\tau}(t)\right) \leq V_{2}\left(E^{\tau}(0)\right) e^{2 \delta t-2(m+1)(\delta+\alpha) \zeta T}, \\
(m+\zeta) T \leq t<(m+1) T ; \\
V_{2}\left(E^{\tau}((m+1) T)\right) \leq V_{2}\left(E^{\tau}(0)\right) e^{2(m+1)(\delta-\delta \zeta-\alpha \zeta) T} .
\end{gathered}
$$


Firstly, we will show the validity of the base case. In case of parameter $m=0$, inequalities (22) and (24) can be reduced as follows:

$$
\begin{aligned}
V_{2}\left(E^{\tau}(t)\right) & \leq V_{2}\left(E^{\tau}(0)\right) e^{-2 \alpha t}, \quad 0 \leq t<\zeta T, \\
V_{2}\left(E^{\tau}(t)\right) & \leq V_{2}\left(E^{\tau}(\zeta T)\right) e^{2 \delta(t-\zeta T)} \\
& \leq V_{2}\left(E^{\tau}(0)\right) e^{2 \delta t-2(\alpha+\delta) \zeta T}, \quad \zeta T \leq t<T,
\end{aligned}
$$

where

$$
\begin{aligned}
& V_{2}\left(E^{\tau}(\zeta T)\right) \leq V_{2}\left(E^{\tau}(0)\right) e^{-2 \alpha \zeta T} \\
& V_{2}\left(E^{\tau}(T)\right) \leq V_{2}\left(E^{\tau}(0)\right) e^{2 \delta T-2(\alpha+\delta) \zeta T} .
\end{aligned}
$$

It can be concluded from the above two inequalities that inequality (25) holds for $m=0$.

Secondly, assuming that inequality (25) is correct for $m=$ $k$, we will show its correctness for $m=k+1$. In fact, if $m=$ $k+1$, inequalities (22) and (24) can be reduced as follows:

$$
\begin{aligned}
V_{2}\left(E^{\tau}(t)\right) \leq & V_{2}\left(E^{\tau^{\top}}((k+1) T)\right) e^{-2 \alpha(t-(k+1) T)} \\
\leq & V_{2}\left(E^{\tau}(0)\right) e^{2(k+1)(\delta+\alpha)(1-\zeta) T-2 \alpha t}, \\
(k+1) T \leq t<(k+1+\zeta) T, & \\
V_{2}\left(E^{\tau}(t)\right) \leq & V_{2}\left(E^{\tau}((k+1+\zeta) T)\right) e^{2 \delta(t-(k+1+\zeta) T)} \\
\leq & V_{2}\left(E^{\tau}(0)\right) e^{2 \delta t-2(k+2)(\delta+\alpha) \zeta T}, \\
& (k+1+\zeta) T \leq t<(k+2) T,
\end{aligned}
$$

where

$$
\begin{aligned}
& V_{2}\left(E^{\tau}((k+1+\zeta) T)\right) \\
& \quad \leq V_{2}\left(E^{\tau}(0)\right) e^{2(k+1)(\delta-\delta \zeta-\alpha \zeta) T-2 \alpha \zeta T}, \\
& V_{2}\left(E^{\tau}((k+2) T)\right) \leq V_{2}\left(E^{\tau}(0)\right) e^{2(k+2)(\delta-\delta \zeta-\alpha \zeta) T} .
\end{aligned}
$$

Then, based on the principle of mathematical induction, we declare that inequality (25) holds for $m=0,1,2, \ldots$.

Combining the monotonic property of the exponential function and the inequality (25), we obtain that

$$
V_{2}\left(E^{\tau}(t)\right) \leq V_{2}\left(E^{\tau}(0)\right) e^{2 m(\delta-\delta \zeta-\alpha \zeta) T}
$$

Noticing condition (iii) of Theorem 4, we can derive that cluster synchronization of the controlled network (2)-(7) is achieved. Hence the proof is completed.

To make Theorem 4 more applicable, we give the following corollary.

Corollary 5. Suppose that Assumption 1 holds, the oscillator network (2)-(7) with the control protocol (9) realizes clusterdelay synchronization if

(i) the constants $\delta-\sigma_{k}-\bar{\lambda}_{k}<0$, where $\bar{\lambda}_{k}$ are the eigenvalues of the symmetric matrix $\left(\bar{A}+\bar{A}^{\top}\right) / 2, k=$ $1,2, \ldots, n$;

(ii) there exists a positive constant $\alpha$ satisfying that

$$
\left(\frac{1}{\zeta}-1\right) \delta \leq \alpha \leq \Theta_{k}-\delta, \quad k=1,2, \ldots, n
$$

It is worth noting that condition (ii) can be simplified into an inequality $\leq \Theta_{k} \zeta, k=1,2, \ldots, n$. The proof of this corollary is not particularly difficult and will not be given here.

3.3. Comparative Studies with Previous Results. In [23], cluster lag synchronization of the undirected networks (1) has been studied by using the intermittent pinning-control method. Enlightened by the design schemes of the controllers with intermittent effect, we proposed the leader-following system (2)-(7) and designed the intermittent pinning controller (9) to realize cluster-delay synchronization.

In order to verify the usefulness of the obtained controller with respect to the previous controllers, we carry out some comparative studies to show the differences from two aspects. The first difference is the definition of cluster lag synchronization with respect to the time delays $\tau_{\varphi_{i}}$, which implies that there holds $\lim _{t \rightarrow+\infty}\left\|x_{i}(t)-s_{\varphi_{i}}\left(t-\tau_{\varphi_{i}}\right)\right\|=0$, where $\dot{s}_{\varphi_{i}}\left(t-\tau_{\varphi_{i}}\right)=f_{\varphi_{i}}\left(s_{\varphi_{i}}\left(t-\tau_{\varphi_{i}}\right)\right), i=1,2, \ldots, N$. In this paper, we proposed the definition of cluster-delay synchronization (Definition 2) in two steps and developed a series of sufficient conditions for both cluster synchronization and clusterdelay synchronization. The second difference is the design schemes of the controllers with intermittent effect. In [23], $\mathrm{Wu}$ and $\mathrm{Fu}$ designed $N$ controllers for each oscillator as follows:

$$
u_{i}(t)= \begin{cases}-\varepsilon \theta_{i}(t)\left(x_{i}(t)-s\left(t-\tau_{\varphi_{i}}\right)\right)-\varepsilon \sum_{j=1}^{N} c_{i j} \Gamma s\left(t-\tau_{\varphi_{i}}\right), & i \in \overline{\mathscr{V}}_{\varphi_{i}} \\ 0, & i \in \mathscr{V}_{\varphi_{i}}-\overline{\mathscr{V}}_{\varphi_{i}},\end{cases}
$$

where the intermittent feedback control gain $\theta_{i}(t)$ was defined by (8). In this paper, we designed $n$ controllers for each cluster in (9) and simplified the complexity of the previous design schemes to some extent.
Based on the aforementioned comparison and analysis, we show the characteristics and advantages of the proposed method with respect to the previous controllers. In our view, the proposed method might serve different purposes in practice. 


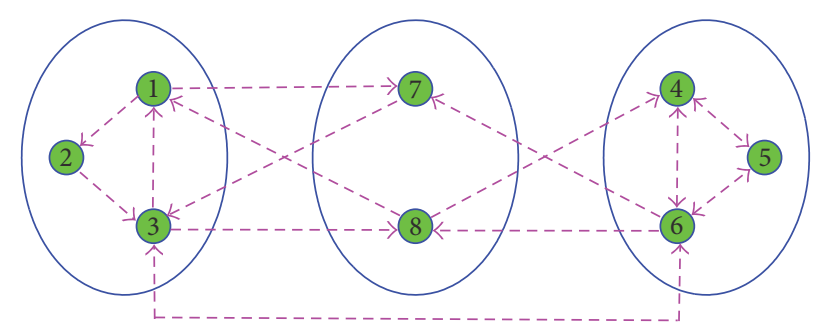

FIGURE 1: Topology structure of a directed network consisting of 8 nodes, which are divided into 3 clusters. If there is a directed link from node $j$ to node $i(j \neq i)$, then $a_{i j}=1$, otherwise $a_{i j}=0$.

\section{Numerical Simulations}

In this section, we carry out some numerical simulations to illustrate the effectiveness of the theoretical results obtained in this paper.

4.1. Numerical Example 1. At first, we consider a directed complex network consisting of 8 nodes separated into three different clusters, the topology of which is shown in Figure 1.

Define connectivity matrix $A$ as an asymmetric matrix with zero row-sums, and the off-diagonal elements $a_{i j}=1$ if the $j$ th node can receive information from the $i$ th node for $j \neq i$; otherwise, define $a_{i j}=0$. Choose the node dynamics of the network as the well-known Chua oscillators; then the oscillator network (2)-(7) with the control protocol (9) can be rewritten as follows:

$$
\begin{aligned}
\dot{x}_{i}= & -D x_{i}+\operatorname{Tg}\left(x_{i}\right)+\sum_{j=1}^{8} a_{i j} x_{j}+u_{i}(t), \\
u_{i}(t)= & \theta_{\varphi(i)}(t)\left[s_{\varphi(i)}(t)-s_{1}\left(t-\tau_{\varphi(i)}\right)\right]-\sum_{j=1}^{8} \widetilde{a}_{i j} x_{j} \\
& -\sigma_{\varphi(i)}\left[x_{i}(t)-s_{1}\left(t-\tau_{\varphi(i)}\right)\right], \\
\dot{s}_{\varphi(i)}(t)= & f\left(s_{\varphi(i)}(t)\right) \\
& -\theta_{\varphi(i)}(t)\left[s_{\varphi(i)}(t)-s_{1}\left(t-\tau_{\varphi(i)}\right)\right],
\end{aligned}
$$

where $x_{i} \in R^{3}, D=I_{3}, g\left(x_{i}\right)=\left(g\left(x_{i 1}\right), g\left(x_{i 2}\right), g\left(x_{i 3}\right)\right)^{\top}, i=$ $1,2, \ldots, 8, g(s)=(|s+1|-|s-1|) / 2$, and

$$
T=\left(\begin{array}{ccc}
1.25 & -3.2 & -3.2 \\
-3.2 & 1.1 & -4.4 \\
-3.2 & 4.4 & 1.0
\end{array}\right) .
$$

It is easy to derive that the nonlinear function $f$ in system (33) satisfies Assumption 1 by choosing the matrix $\Delta=5.5685 I_{3}$. Then, one can choose $\delta=5.5685, \sigma_{1}=10$, $\sigma_{2}=12, \sigma_{3}=14$ such that condition (i) of Theorem 4 holds, and choose $\alpha=4.4, \Theta_{1}=\Theta_{2}=\Theta_{3}=10$ such that condition (ii) holds. By taking $T=1$, it can be verified that the feedback control width $\zeta=0.557$ satisfies condition (iii) of Theorem 4 .

Choose the initial conditions randomly; Figure 2 is plotted to show the evolutions of the state variable of the 8 nodes and the cluster errors. From (a), (b), and (c), one can see that the evolutions of the nodes in the same cluster synchronize with each other. And (d) shows more clearly that the cluster errors $e_{i}(t)=x_{i}(t)-s_{\varphi(i)}(t)$ tend to zero. Therefore, Figure 2 illustrates that cluster-delay synchronization is achieved under the conditions of Theorem 4.

Next, we keep all the parameters unchanged except the feedback control gains decreasing from $\sigma_{1}=10, \sigma_{2}=12$, $\sigma_{3}=14$ to $\sigma_{1}=\sigma_{2}=\sigma_{3}=2$. Then the conditions of Theorems 3 and 4 cannot be satisfied. The time evolutions of the Chua oscillators $x_{i 1}(t), x_{i 2}(t), x_{i 3}(t)(i=1,2, \ldots, 8)$ in the network (33) are plotted in Figure 3. One can see clearly that the evolutions of nodes 7 and 8 do not synchronize with each other though they are in the same cluster. Therefore, neither cluster synchronization nor cluster-delay synchronization is achieved.

4.2. Numerical Example 2. In the following numerical simulations, we consider an undirected network consisting of $100 x_{\cdot 2}$-coupled Lorenz systems separated into two clusters. The network topology is shown in Figure 4. If there is an undirected link between node $j$ and node $i(j \neq i)$, then $a_{i j}=a_{j i}=1$; otherwise $a_{i j}=a_{j i}=0$. by

The uncoupled Lorenz system $\dot{x}_{i}=f\left(x_{i}, t\right)$ is described

$$
\begin{aligned}
& \dot{x}_{i 1}=\sigma\left(x_{i 2}-x_{i 1}\right), \\
& \dot{x}_{i 2}=r x_{i 1}-x_{i 2}-x_{i 1} x_{i 3}, \\
& \dot{x}_{i 3}=-b x_{i 3}+x_{i 1} x_{i 2},
\end{aligned}
$$

where $\sigma=10, r=28, b=8 / 3$. According to [30], there exists a positive constant $\delta$ such that Assumption 1 holds. Then, one can choose appropriate values for other parameters in the network (2)-(7) with the controller (9) such that the conditions of Theorem 4 are satisfied. The time evolutions of the 100 Lorenz systems are shown in Figure 5. As indicated by Figure 5, the first fifty nodes fall into the same cluster, and the rest of the nodes fall into another cluster. All the nodes in the same cluster behave in the same synchronous fashion, but nodes in different clusters follow distinct time evolutions with time delays. Therefore, cluster-delay synchronization has been realized in the network consisting of $100 x_{\cdot 2}$-coupled Lorenz systems. 

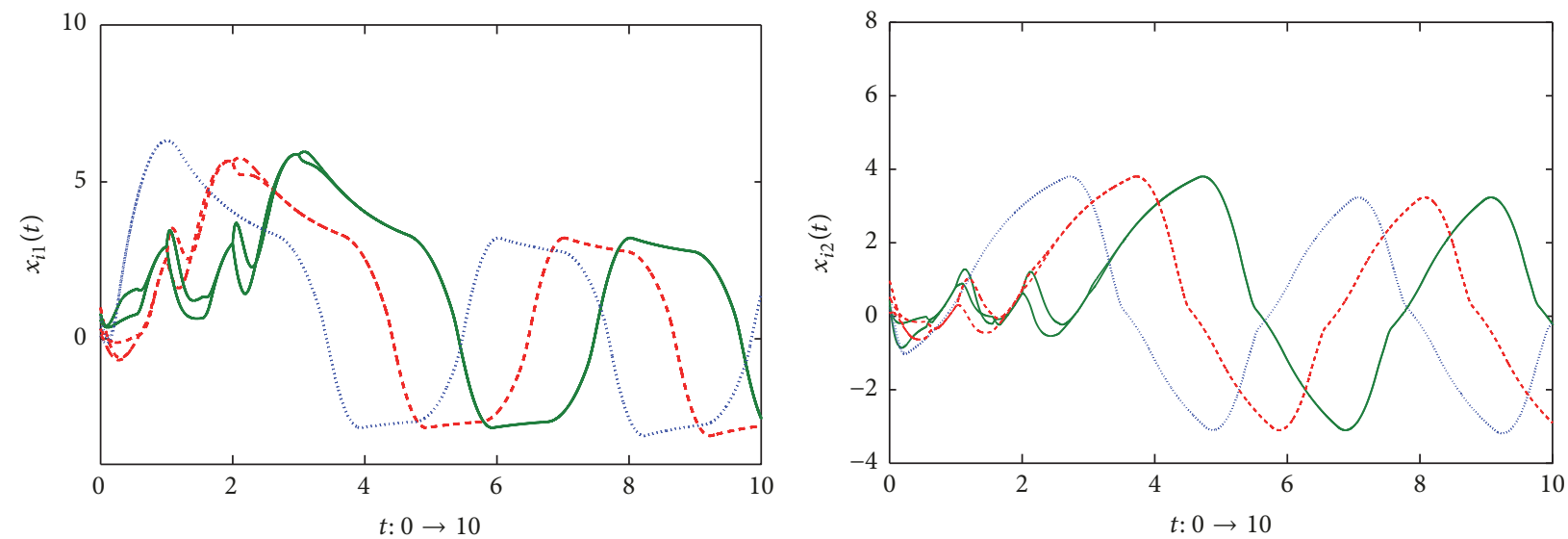

$\begin{aligned} & x_{11}, x_{21}, x_{31} \\ \ldots & x_{41}, x_{51}, x_{61} \\ - & x_{11}, x_{81}\end{aligned}$

(a)

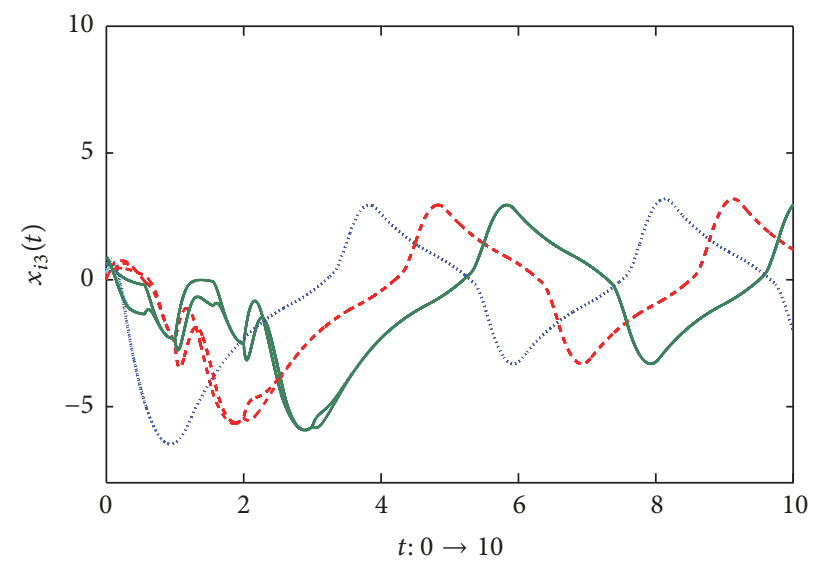

$\begin{aligned} & x_{13}, x_{23}, x_{33} \\ \ldots . & x_{43}, x_{53}, x_{63} \\ - & x_{73}, x_{83}\end{aligned}$

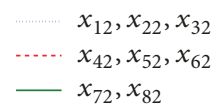

(b)

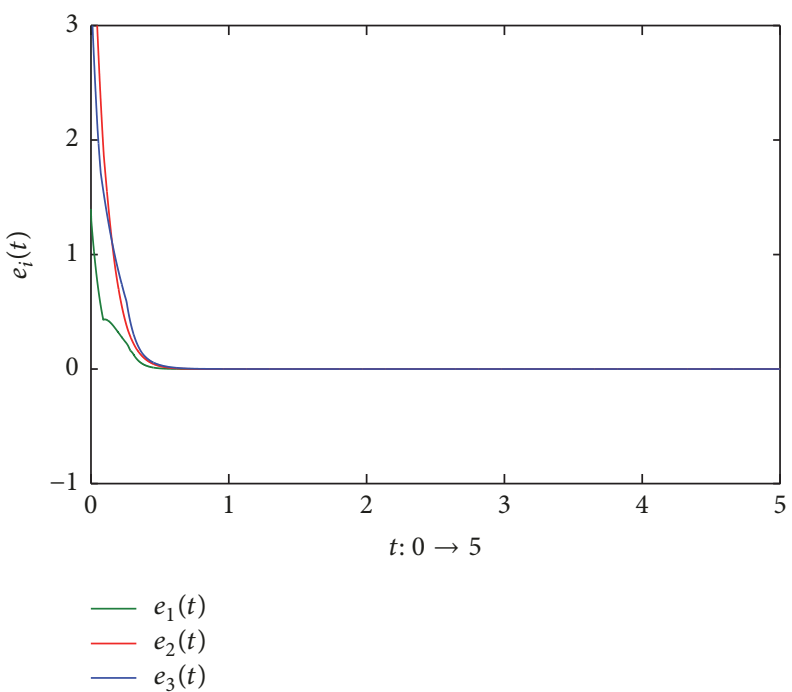

(d)

FIGURE 2: Cluster-delay synchronization of the network (33). (a), (b), and (c) show the time evolutions of the state variable of the 8 nodes splitting into three different clusters, and $(\mathrm{d})$ describes the time evolutions of the cluster errors.
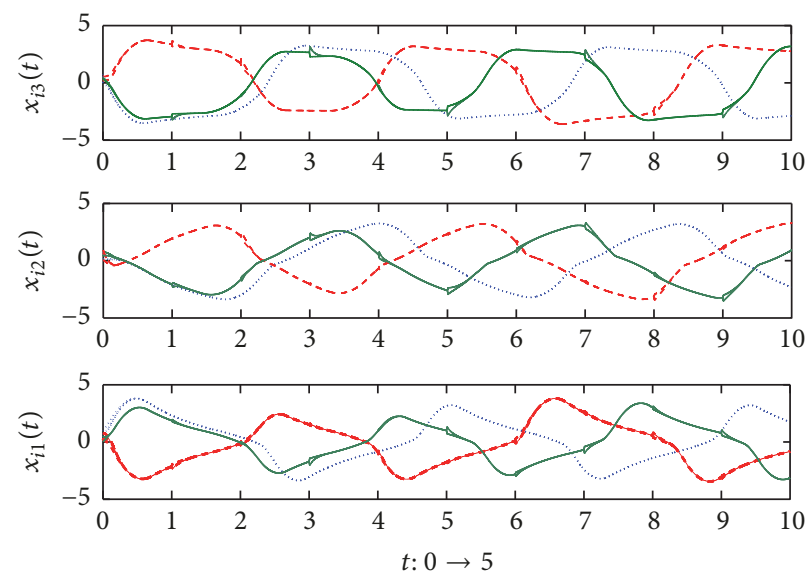

FIgure 3: Time evolutions of the Chua oscillators $x_{i 1}(t), x_{i 2}(t), x_{i 3}(t)(i=1,2, \ldots, 8)$ in the network (33) with the feedback control gains $\sigma_{1}=\sigma_{2}=\sigma_{3}=2$. 


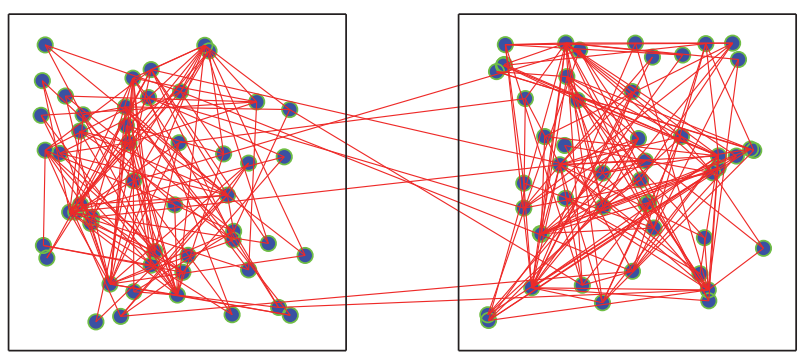

FIGURE 4: Topology structure of the undirected network consisting of 100 nodes, which are separated into two equal clusters. If there is an undirected link between node $j$ and node $i(j \neq i)$, then $a_{i j}=$ $a_{j i}=1$, otherwise $a_{i j}=a_{j i}=0$.
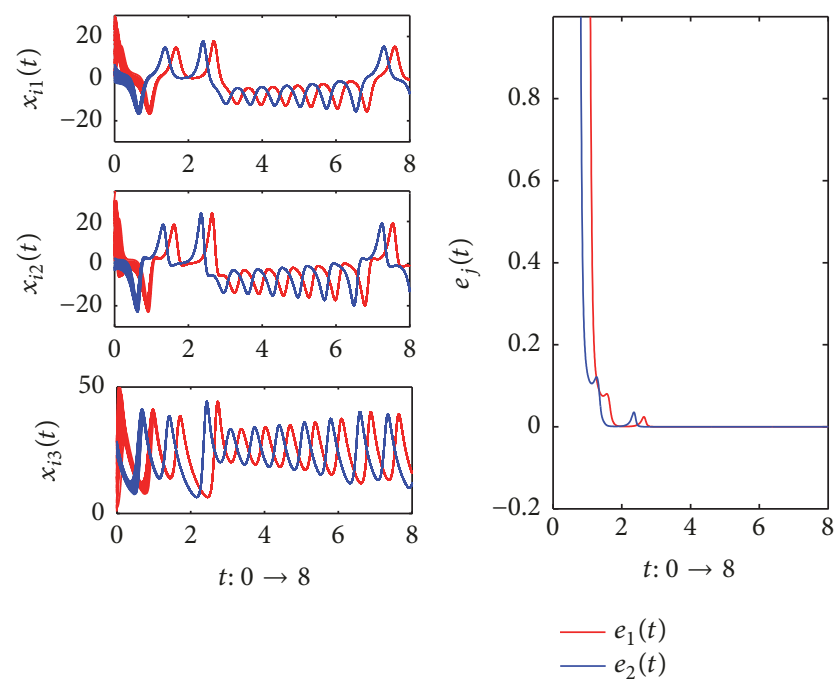

Figure 5: Time evolutions of the $x_{\cdot 2}$-coupled Lorenz systems $x_{i 1}(t)$, $x_{i 2}(t), x_{i 3}(t)(i=1,2, \ldots, 100)$ in the network (2)-(7) with the controller (9).

\section{Conclusions}

In this paper, the cluster-delay synchronization problem of a directed network with cluster structure has been discussed. First, a control protocol with intermittent effect has been presented to realize cluster synchronization via periodically intermittent control. The proposed control methods can be applied to discuss many real-world networks with intermittent effect. Second, we extended the criterion on cluster synchronization to the cluster-delay synchronization problem, which implies that the oscillators in the following clusters track the trajectory of those in the leader clusters with different time delays. Finally, we presented two delayed dynamical networks as illustrative examples and carry out some simulated results to show the feasibility of the proposed control methods.

\section{Conflicts of Interest}

The authors declare that they have no conflicts of interest regarding the publication of this paper.

\section{Acknowledgments}

This work was supported by NNSF of China (11447005, 11562006, 61703193, and 61663006), Jiangsu Planned Projects for Postdoctoral Research Funds (1701017A), NSF of Shandong Province (ZR2016JL021), NSF of Guangxi Province (2016GXNSFDA380031), the Key Research and Development Project of Shandong Province of China (2017GGX10143), and the Key Research and Development Project of Linyi City of China (2017GGH009).

\section{References}

[1] J. Guckenheimer, "Sensitive dependence to initial conditions for one-dimensional maps," Communications in Mathematical Physics, vol. 70, no. 2, pp. 133-160, 1979.

[2] L. M. Pecora and T. L. Carroll, "Synchronization in chaotic systems," Physical Review Letters, vol. 64, no. 8, pp. 821-824, 1990.

[3] L. M. Pecora and T. L. Carroll, "Master stability functions for synchronized coupled systems," Physical Review Letters, vol. 80, no. 10, pp. 2109-2112, 1998.

[4] V. N. Belykh, I. V. Belykh, and M. Hasler, "Connection graph stability method for synchronized coupled chaotic systems," Physica D: Nonlinear Phenomena, vol. 195, no. 1-2, pp. 159-187, 2004.

[5] W. Lu and T. Chen, "New approach to synchronization analysis of linearly coupled ordinary differential systems," Physica D: Nonlinear Phenomena, vol. 213, no. 2, pp. 214-230, 2006.

[6] C.-D. Yang, J.-L. Qiu, and H.-B. He, "Exponential synchronization for a class of complex spatio-temporal networks with space-varying coefficients," Neurocomputing, vol. 151, pp. 401407, 2015.

[7] Y.-P. Luo, L. Shu, and B.-F. Zhou, "Global exponential synchronization of nonlinearly coupled complex dynamical networks with time-varying coupling delays," Complexity, vol. 2017, Article ID 7850958, 2017.

[8] Z. Wu and X. Fu, "Cluster projective synchronization between community networks with nonidentical nodes," Physica A: Statistical Mechanics and its Applications, vol. 391, no. 23, pp. 6190-6198, 2012.

[9] J. Guan, "Adaptive modified generalized function projection synchronization between integer-order and fractional-order chaotic systems," Optik - International Journal for Light and Electron Optics, vol. 127, no. 10, pp. 4211-4216, 2016.

[10] H. Liu, W. Sun, and G. Al-mahbashi, "Parameter identification based on lag synchronization via hybrid feedback control in uncertain drive-response dynamical networks," Advances in Difference Equations, vol. 2017, no. 1, article no. 122, 2017.

[11] J. Zhang, Z. Ma, and G. Chen, "Robustness of cluster synchronous patterns in small-world networks with inter-cluster co-competition balance," Chaos: An Interdisciplinary Journal of Nonlinear Science, vol. 24, no. 2, article no. 023111, 2014.

[12] A. Hu, J. Cao, M. Hu, and L. Guo, "Cluster synchronization in directed networks of non-identical systems with noises via random pinning control," Physica A: Statistical Mechanics and its Applications, vol. 395, pp. 537-548, 2014.

[13] C. Yang, J. Cao, T. Huang, J. Zhang, and J. Qiu, "Guaranteed cost boundary control for cluster synchronization of complex spatio-temporal dynamical networks with community structure," Science China Information Sciences, vol. 61, no. 5, article no. 052203, pp. 1-13, 2018. 
[14] S. Cai, Q. Jia, and Z. Liu, "Cluster synchronization for directed heterogeneous dynamical networks via decentralized adaptive intermittent pinning control," Nonlinear Dynamics, vol. 82, no. 1-2, pp. 689-702, 2015.

[15] H. Su, Z. Rong, M. Z. Q. Chen, X. Wang, G. Chen, and H. Wang, "Decentralized adaptive pinning control for cluster synchronization of complex dynamical networks," IEEE Transactions on Systems, Man, and Cybernetics, Part B: Cybernetics, vol. 43, no. 1, pp. 394-399, 2013.

[16] J. Lu, J. Kurths, J. Cao, N. Mahdavi, and C. Huang, "Synchronization control for nonlinear stochastic dynamical networks: Pinning impulsive strategy," IEEE Transactions on Neural Networks and Learning Systems, vol. 23, no. 2, pp. 285-292, 2012.

[17] J. Guan and K. Wang, "Sliding mode control and modified generalized projective synchronization of a new fractionalorder chaotic system," Mathematical Problems in Engineering, vol. 2015, Article ID 941654, 2015.

[18] W. Yu, G. Chen, J. Lu, and J. Kurths, "Synchronization via pinning control on general complex networks," SIAM Journal on Control and Optimization, vol. 51, no. 2, pp. 1395-1416, 2013.

[19] R. Rakkiyappan and N. Sakthivel, "Cluster synchronization for T-S fuzzy complex networks using pinning control with probabilistic time-varying delays," Complexity, vol. 21, no. 1, pp. 59-77, 2015.

[20] H.-X. Hu, Q. Xuan, W. Yu, C.-G. Zhang, and G. Xie, "Secondorder consensus for heterogeneous multi-agent systems in the cooperation-competition network: a hybrid adaptive and pinning control approach," Nonlinear Analysis: Hybrid Systems, vol. 20, pp. 21-36, 2016.

[21] X. Chen, J. H. Park, J. Cao, and J. Qiu, "Adaptive synchronization of multiple uncertain coupled chaotic systems via sliding mode control," Neurocomputing, vol. 273, pp. 9-21, 2018.

[22] S. Cai, P. Zhou, and Z. Liu, "Pinning synchronization of hybridcoupled directed delayed dynamical network via intermittent control," Chaos: An Interdisciplinary Journal of Nonlinear Science, vol. 24, no. 3, Article ID 033102, 2014.

[23] Z. Wu and X. Fu, "Cluster lag synchronisation in community networks via linear pinning control with local intermittent effect," Physica A: Statistical Mechanics and its Applications, vol. 395, pp. 487-498, 2014.

[24] J. Feng, P. Yang, and Y. Zhao, "Cluster synchronization for nonlinearly time-varying delayed coupling complex networks with stochastic perturbation via periodically intermittent pinning control," Applied Mathematics and Computation, vol. 291, pp. 52-68, 2016.

[25] C. Yu, J. Qin, and H. Gao, "Cluster synchronization in directed networks of partial-state coupled linear systems under pinning control," Automatica, vol. 50, no. 9, pp. 2341-2349, 2014.

[26] W. Sun, S. Wang, and J. Zhang, "Counting spanning trees in prism and anti-prism graphs," Journal of Applied Analysis and Computation, vol. 6, no. 1, pp. 65-75, 2016.

[27] Z. Ma, Y. Wang, and X. Li, "Cluster-delay consensus in firstorder multi-agent systems with nonlinear dynamics," Nonlinear Dynamics, vol. 83, no. 3, pp. 1303-1310, 2016.

[28] T. Chen, X. Liu, and W. Lu, "Pinning complex networks by a single controller," IEEE Transactions on Circuits and Systems I: Regular Papers, vol. 54, no. 6, pp. 1317-1326, 2007.

[29] Q. Song and J. Cao, "On pinning synchronization of directed and undirected complex dynamical networks," IEEE Transactions on Circuits and Systems I: Regular Papers, vol. 57, no. 3, pp. 672-680, 2010.
[30] J.-B. Zhang, Z.-J. Ma, and G. Zhang, "Control schemes for synchronizing two subnetworks with weak couplings," Chinese Physics B, vol. 23, no. 1, Article ID 010507, 2013. 


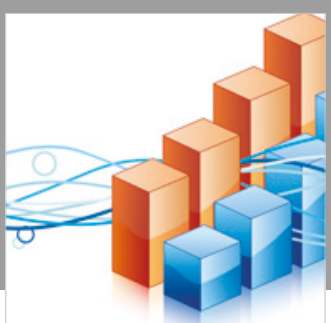

Advances in

Operations Research

\section{-n-m}
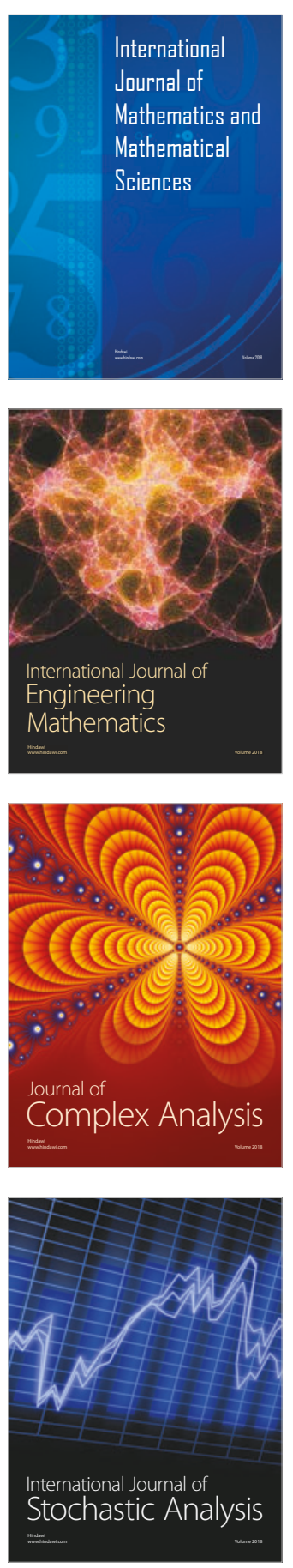
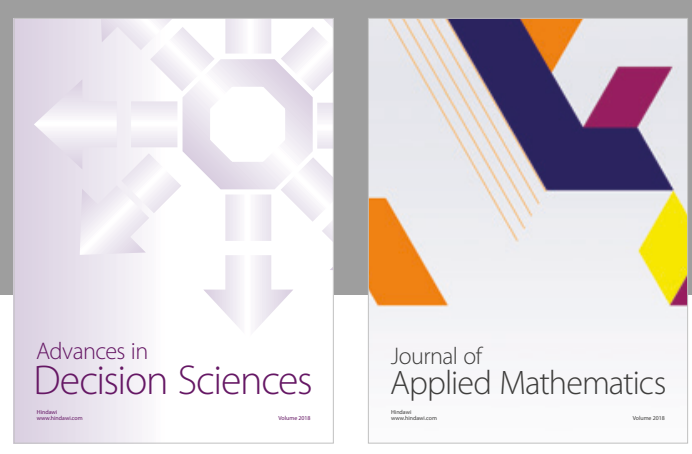

Journal of

Applied Mathematics
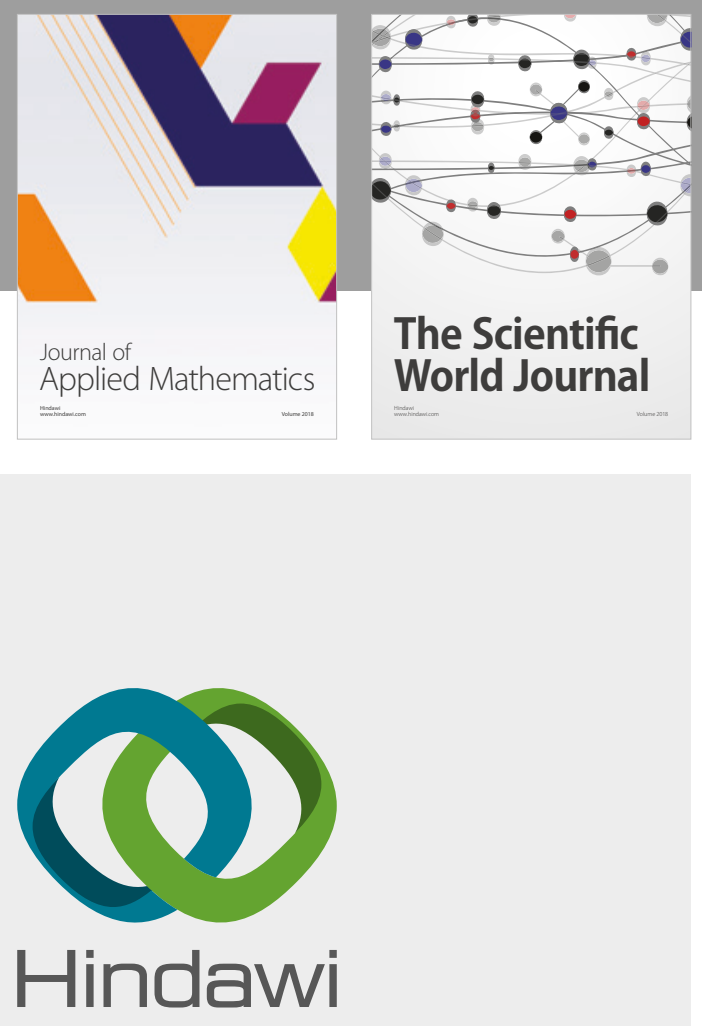

Submit your manuscripts at

www.hindawi.com

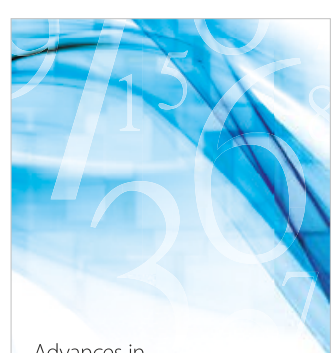

Advances in
Numerical Analysis
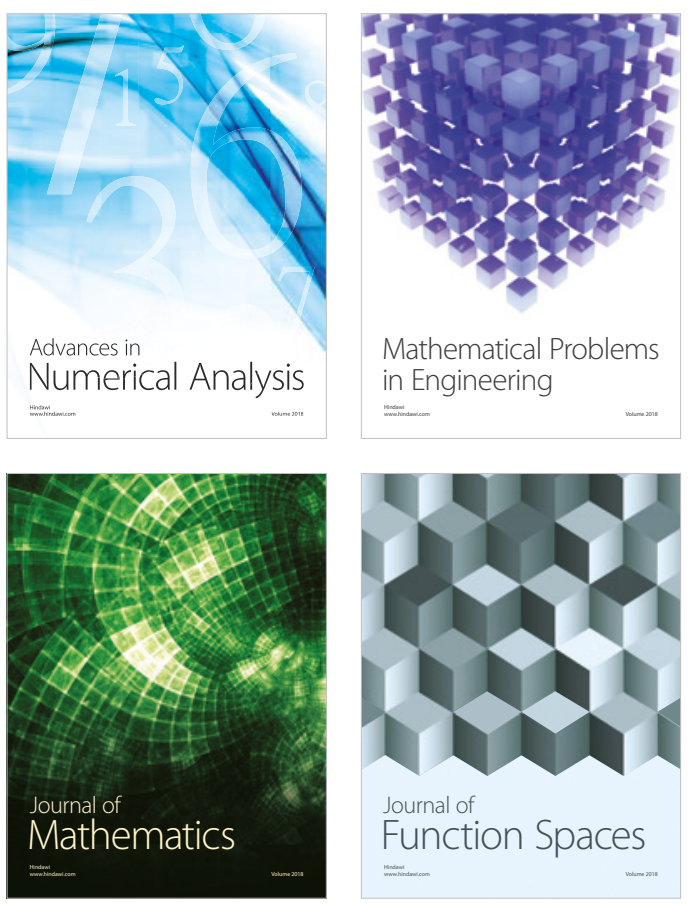

Mathematical Problems in Engineering

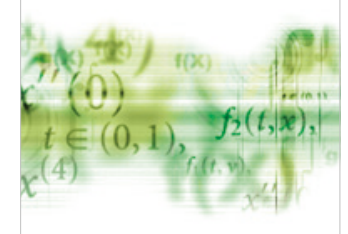

International Journal of

Differential Equations

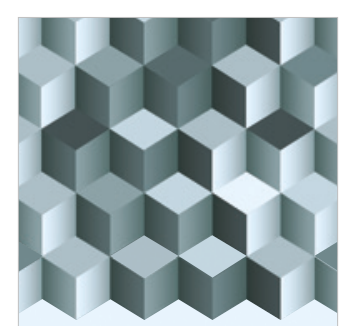

Journal of

Function Spaces
The Scientific

World Journal

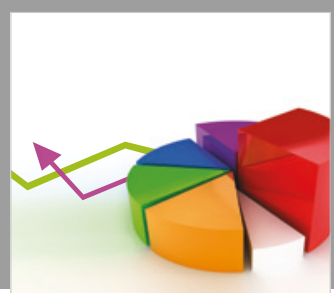

Journal of

Probability and Statistics
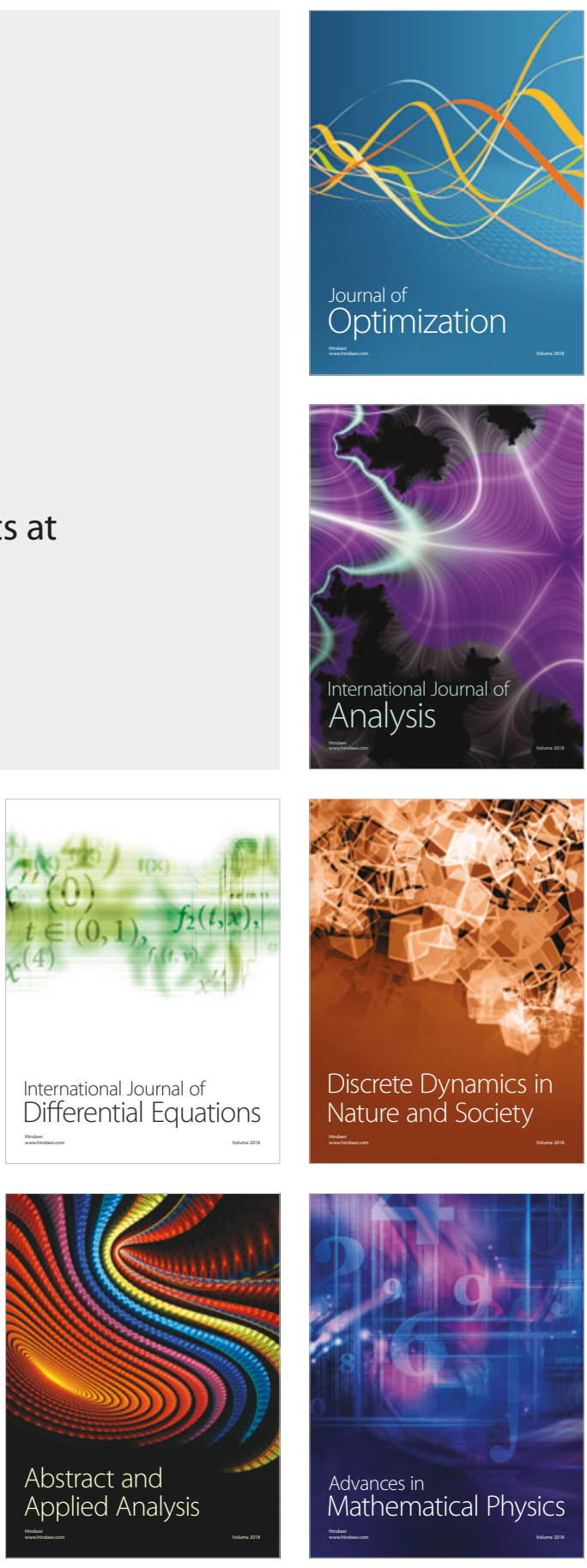